\section{Top universal composite ten years running}

Trycare Ltd, the UK's fastest growing dental dealer, has announced that Estelite Sigma Quick has been awarded the Dental Advisor Top Product Universal Composite for the tenth year running.

Featuring Tokuyama's patented RAP monomer and aesthetic 'Pearl' technology, Estelite Sigma Quick delivers an extended working time yet cures in only ten seconds. There is also less residual monomer and minimal after cure colour change for long term aesthetic satisfaction.

In addition, Estelite Sigma Quick offers what the company calls 'miracle' shade matching. Because of its unique aesthetic 'Pearls' it offers inherent shade mimicking so that, in most cases, just one shade will blend perfectly with the natural teeth, leaving invisible margins and undetectable restorations.

These 'Pearls' also facilitate outstanding ability to polish that lasts, allied to high wear resistance and very low abrasion of the opposing teeth.

Other Trycare products to receive the Dental Advisor's Top Product for 2019 status were Tokuyama Sofliner Tough S in the denture reline material classification and Garrison Tight 3D Fusion in the Sectional Matrix category. Tokuyama Shield Force Plus received the Preferred Product Award Winner in the desensitiser category.

More information is available by calling Trycare Ltd on 01274 885544 or visiting www.trycare.co.uk/estelite.

\section{Aesthetics and performance combined}

Want to offer your patients an effective orthodontic solution that also looks good? Enter a new generation of beautiful aesthetics by choosing Clarity ADVANCED brackets from 3M Oral Care.

Featuring a dome-shaped design for increased patient comfort, Clarity ADVANCED brackets take orthodontic treatment to the next level of discretion. This ensures that patients feel confident so they can keep smiling even when the brackets are in place.

Easy to match to a variety of tooth shades, the brackets are also stain-resistant, meaning patients will not have to worry about any discolouration throughout the course of treatment.

Give your patients an aesthetic choice with Clarity ADVANCED brackets from 3M Oral Care.

For more information, call 08458734066 or visit http:// solutions.3m.co.uk/wps/portal/3M/en_GB/orthodontics_EU/ Unitek/. 3M and Clarity ADVANCED are trademarks of the 3M Company.
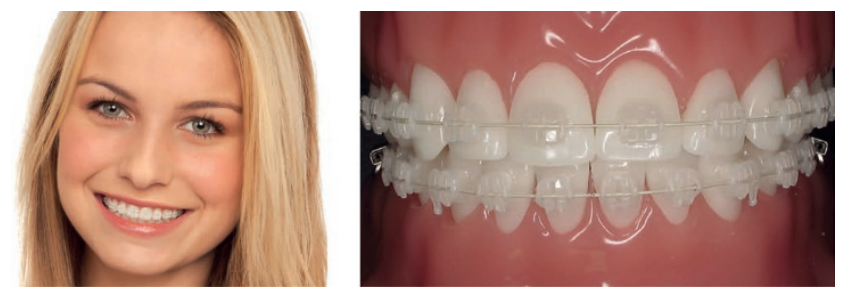

\section{State of the art new products for layering, milling and bonding}

The latest state of the art products from Shofu Dental will be on show at the International Dental Show (IDS 2019) taking place from 12 to 16 March 2019 in Cologne, Germany.

At the dental business summit, Shofu will be in Hall 11.3 (Booth G010/J019) for the first time with a new concept including not only product presentation and information, but also communication and customer connectivity. The booth area of more than 240 square metres will provide sufficient room for new, extended and improved products, as well as a lively exchange of views with dentists, dental technicians and distribution partners.

Shofu will focus on direct and CAD/CAM restorative materials, polishing, porcelain systems, and digital dental photography.

Whether for the newly developed BeautiBond Universal multifunctional adhesive system, the innovative Shofu Disk ZR Lucent multilayer zirconia disks, the recently launched OneGloss $M$ polishers, the new Beautifil Flow Plus X restorative in two viscosities, or the popular EyeSpecial C-III camera, a visit to Shofu's booth at the IDS will be worthwhile.

Shofu is increasingly focused on providing practical information and tips to users in both analogue and digital formats hence a variety of new instructions for SSE and electronic tools will be available at the IDS.

Martin Hesselmann, European Managing Director of Shofu, said: 'Communication in both ways, rather than one way is the motto of our new IDS booth concept. We not only wish to send messages to our customers, but also to enter into a dialogue with them and initiate a mutual exchange of views for new, forward-thinking solutions.'

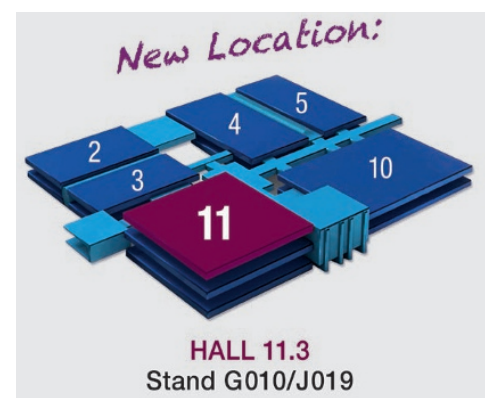

\section{Helping you to help your patients}

Whether a particularly complex case demands more than your practice is equipped for, or you simply want a second opinion, BPI Dental in Birmingham is an ideal partner for referrals.

BPI Dental has invested in a high quality CBCT scanner that can produce excellent 3D imagery, illuminating the details and complexities that traditional X-rays can miss.

BPI Dental says it will not leave practitioners in the dark. A full report, periodontal chart and X-rays will be sent to them, with radiographs available upon request.

For more information on the referral services available from Birmingham Periodontal \& Implant (BPI) Dental, visit www. bpidental.co.uk or call 01214273210 or email info@bpidental.co.uk.

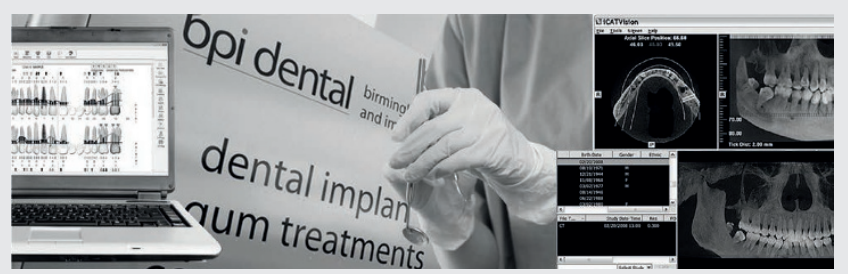

